\title{
Sedentary Behavior and Physical Function Decline in Older Women: Findings from the Women's Health Initiative
}

\author{
Rebecca Seguin, ${ }^{1,2}$ Michael LaMonte, ${ }^{3}$ Lesley Tinker, ${ }^{1}$ Jingmin Liu, ${ }^{1}$ Nancy Woods, ${ }^{4}$ \\ Yvonne L. Michael, ${ }^{5}$ Cheryl Bushnell, ${ }^{6}$ and Andrea Z. LaCroix ${ }^{1,2}$ \\ ${ }^{1}$ Fred Hutchinson Cancer Research Center, Seattle, WA 98109, USA \\ ${ }^{2}$ Group Health Research Institute, Seattle, WA 98101, USA \\ ${ }^{3}$ Department of Social and Preventive Medicine, University at Buffalo, Buffalo, NY 14214, USA \\ ${ }^{4}$ School of Nursing, University of Washington, Seattle, WA 98195, USA \\ ${ }^{5}$ Drexel University School of Public Health, Philadelphia, PA 19102, USA \\ ${ }^{6}$ Department of Neurology, Wake Forest University Health Sciences, Winston Salem, NC 27157, USA
}

Correspondence should be addressed to Rebecca Seguin, rebeccaseguin@hotmail.com

Received 21 January 2012; Accepted 6 March 2012

Academic Editor: Wojtek Chodzko-Zajko

Copyright (c) 2012 Rebecca Seguin et al. This is an open access article distributed under the Creative Commons Attribution License, which permits unrestricted use, distribution, and reproduction in any medium, provided the original work is properly cited.

\begin{abstract}
Sedentary behavior is associated with deleterious health outcomes. This study evaluated the association between sedentary time and physical function among postmenopausal women in the Women's Health Initiative Observational Study. Data for this prospective cohort study were collected between 1993-1998 (enrollment) and 2009, with an average of 12.3 follow-up years. Analyses included 61,609 women (aged 50-79 years at baseline). Sedentary time was estimated by questionnaire; physical function was measured using the RAND SF-36 physical function scale. Mixed-model analysis of repeated measures was used to estimate the relationship of sedentary time exposures and changes in physical function adjusting for relevant covariates. Compared to women reporting sedentary time of $\leq 6$ hours/day, those with greater amounts of sedentary time ( $>6-8$ hours/day, $>8-11$ hours/day, $>11$ hours/day) reported lower physical function between baseline and follow up (coefficient $=-0.78, \mathrm{CI}=-0.98,-0.57,-1.48, \mathrm{CI}=$ $-1.71,-1.25,-3.13$, and $\mathrm{CI}=-3.36,-2.89$, respectively $P<0.001)$. Sedentary time was strongly associated with diminished physical function and most pronounced among older women and those reporting the greatest sedentary time. Maintaining physical function with age may be improved by pairing messages to limit sedentary activities with those promoting recommended levels of physical activity.
\end{abstract}

\section{Introduction}

Participation in regular physical activity confers many health benefits, including reduced risk of heart disease, hypertension, stroke, dyslipidemia, obesity, diabetes, osteoporosis, certain cancers, and all-cause mortality [1-7]. Physical activity also improves skeletal muscle function and may ameliorate symptoms of arthritis, depression, and sleep disturbances in older women [8-11]. In general, greater quantity and intensity of exercise have increased benefits, although studies have demonstrated benefits with less-frequent, lowerintensity activities over short bouts [6]. Consistent with this evidence, prolonged exposure to sedentary time has been associated with increased risk of many of the aforementioned diseases and conditions [12-14].

In much of the research to date, sedentary time is classified based on lack of self-reported physical activity, as compared to asking about or measuring actual time spent sitting or lying. Specifically, an assessment of sedentary time may include time spent in behaviors such as screen viewing (i.e., watching television or working at a computer) and other daily episodes of sitting or lying to work, eat, read, or socialize. Studies examining associations of sedentary time with measures of general health or with risk of disability and disease are growing but still quite limited. Of those available, greater sedentary time has been shown to impart 
deleterious physiologic effects [15] and increased risk for obesity, metabolic syndrome, and type 2 diabetes [1618]. Additionally, research from the Canada Fitness Survey examined data from 17,013 men and women, in which they observed a dose-response relationship between sitting time and mortality from all causes and cardiovascular disease after controlling for usual physical activity habits [19].

A related area of investigation is to understand the effects of sedentary time on physical function (PF) - including strength, mobility, and self-care-in aging populations. A recent report by Buman and colleagues examined objective accelerometer measures of physical activity in older adults and found that even low intensity physical activity was associated with health benefits, including greater PF [20]. While research has established the importance of regular physical activity participation for maintaining PF in older adults $[8,21]$, more data are needed to understand whether exposure to greater sedentary time after menopause is an independent risk factor for decline in PF at later ages, and to what extent other factors may influence this association. This study utilized data from the Women's Health Initiative Observational Study and Extension Studies (WHI-OSES) to examine these relationships in a well-characterized cohort of older postmenopausal women.

\section{Methods}

2.1. Purpose. This study aims to understand the relationship between sedentary time and decline in self-reported physical function among women in the WHI-OSES. We hypothesized that greater amounts of daily sedentary time would be associated prospectively with lower physical function scores.

2.2. Study Population. The Women's Health Initiative (WHI) includes observational and randomized controlled intervention studies conducted among postmenopausal women at 40 clinical centers across the United States. The design and population for those studies have been previously described [22-24]. This study focuses on participants from the Women's Health Initiative Observational Study (WHIOS) who continued with Women's Health Initiative Extension Study, herein WHI-OSES. Briefly, the WHI-OS with continued follow up in the WHI-OSES is a large prospective study conducted to investigate morbidity and mortality in aging women. The WHI-OS participants were recruited from 1993-1998 and included 93,676 racially and ethnically diverse women aged 50-79 years. Data from participants in WHI-OS were collected at baseline and follow up using inperson interviews, physical measurements, blood samples, and self-report questionnaires.

The WHI Extension Study (WHI-ES) was designed to collect an additional five years of follow up data (20052010) among all women in WHI, including the randomized trial participants, to describe the long-term effects of the interventions as well as continue to optimize the vast scientific assets of the WHI-OS longitudinal dataset. Eligible WHI-OS participants were mailed information about the extension study at closeout of the original parent study (2004-2005) and asked to consider providing written consent for extended follow up and to update their personal contact information.

Among the 93,676 WHI-OS women, 86,744 (92.6\%) were eligible for the WHI-ES (women deemed ineligible were deceased, $N=5,463$ or had lost contact, $N=1,469$ ). Of the eligible women, 63,231 (72.9\%) consented and were enrolled. Of these $63,231 \mathrm{WHI}$-OSES women, 61,609 provided data on baseline sedentary time exposure and PF required for these analyses.

2.3. Physical Function. Physical function was assessed at baseline (1993-1998), year 3 (1996-2001), and each year of the WHI-ES (ES years $1-5 ; 2005-2010$ ) using the ten PF items from the RAND SF-36 scale, a well-validated measure of self-reported PF $[25,26]$. The PF scale includes items that measure whether current health limits $\mathrm{PF}$ in four general domains (moderate/vigorous activities (2 items), strength (4 items), walking abilities (3 items), and self-care ( 1 item)). Scoring is from 0-100; a higher score indicates superior PF.

2.4. Sedentary Time. Sedentary time at baseline and at Year 3 was assessed using questions that asked the respondent to determine total hours spent sitting (e.g., at work, eating, driving, riding, watching TV, talking) in a usual given day, and the total hours spent lying down in a usual typical day (e.g., sleeping, trying to sleep, watching TV, resting, napping). Total sedentary time is calculated by combining the reported hours of sitting and lying time and then subtracting reported hours sleeping (asked separately from the question on lying time).

2.5. Covariates. Additional baseline covariates of interest included age, race/ethnicity, income, education, body mass index (BMI, $\left.\mathrm{kg} / \mathrm{m}^{2}\right)$, physical activities, self-reported health status, number of chronic diseases, smoking, alcohol use, hypertension, treated diabetes, history of stroke, history of coronary heart disease (CHD), history of congestive heart failure (CHF), history of chronic obstructive pulmonary disease (COPD), history of falls, history of cancer, history of arthritis, hormone use, living alone, number of falls in past 12 months, depressed mood, and activity of daily living disability. These variables were chosen due to their potential relationship with PF decline. In addition, models were adjusted for usual recreational physical activity. Physical activity was assessed by a questionnaire that captured duration and frequency of walking and other recreational activities according to intensity groups (mild, moderate, strenuous/very hard). Weekly energy expenditure (MET-hours/ week) was computed by summing the product of duration and intensity for each physical activity, with intensity defined in metabolic equivalents (METs) obtained from a standardized classification system [27].

2.6. Statistical Approach. Baseline descriptive characteristics were examined by quartiles of sitting and total sedentary time. Since sample characteristics were similar according to sitting time and sedentary time, we present these data only by total sedentary time in Table 1 . Two-sided $P$-values comparing these baseline characteristics across quartiles were 
TABLE 1: Baseline characteristics of participants by total sedentary time, Women's Health Initiative Observational Study and Extension Study (WHI-OSES), $(N=61,609)$.

\begin{tabular}{|c|c|c|c|c|c|}
\hline \multirow{3}{*}{ Characteristic } & \multicolumn{4}{|c|}{ Total sedentary time (hours per day) ${ }^{1}$} & \multirow{3}{*}{$P$ value ${ }^{2}$} \\
\hline & Q1 ( $\leq 6 \mathrm{hrs} /$ day $)$ & Q2 (>6-8 hrs/day) & Q3 (>8-11 hrs/day) & Q4 (>11 hrs/day) & \\
\hline & $n(\%)$ & $n(\%)$ & $n(\%)$ & $n(\%)$ & \\
\hline All & $19364(31.53 \%)$ & $12316(20.06 \%)$ & $15958(25.99 \%)$ & $13769(22.42 \%)$ & \\
\hline \multicolumn{6}{|l|}{ Age group at screening, years } \\
\hline $50-59$ & $6010(31.04 \%)$ & $3622(29.41 \%)$ & $5622(35.23 \%)$ & $6049(43.93 \%)$ & \multirow{3}{*}{$<.0001$} \\
\hline $60-69$ & $9213(47.58 \%)$ & $5927(48.12 \%)$ & $7097(44.47 \%)$ & $5623(40.84 \%)$ & \\
\hline $70-79$ & $4141(21.39 \%)$ & $2767(22.47 \%)$ & $3239(20.30 \%)$ & $2097(15.23 \%)$ & \\
\hline \multicolumn{6}{|l|}{ Ethnicity } \\
\hline White & $16507(85.25 \%)$ & $11103(90.15 \%)$ & $14355(89.95 \%)$ & $12301(89.34 \%)$ & \multirow{6}{*}{$<.0001$} \\
\hline Black & $1387(7.16 \%)$ & $564(4.58 \%)$ & $743(4.66 \%)$ & $744(5.40 \%)$ & \\
\hline Hispanic & $694(3.58 \%)$ & $264(2.14 \%)$ & $300(1.88 \%)$ & $248(1.80 \%)$ & \\
\hline American Indian & $84(0.43 \%)$ & $36(0.29 \%)$ & $50(0.31 \%)$ & $34(0.25 \%)$ & \\
\hline Asian/Pacific Islander & $410(2.12 \%)$ & $213(1.73 \%)$ & $326(2.04 \%)$ & $300(2.18 \%)$ & \\
\hline Unknown & $282(1.46 \%)$ & $136(1.10 \%)$ & $184(1.15 \%)$ & $142(1.03 \%)$ & \\
\hline \multicolumn{6}{|l|}{ Income } \\
\hline$<\$ 20,000$ & $2513(13.94 \%)$ & $1327(11.51 \%)$ & $1671(11.05 \%)$ & $1387(10.59 \%)$ & \multirow{5}{*}{$<.0001$} \\
\hline$\$ 20,000-\$ 34,999$ & $3920(21.74 \%)$ & $2604(22.59 \%)$ & $3255(21.52 \%)$ & $2775(21.19 \%)$ & \\
\hline$\$ 35,000-\$ 49,999$ & $3724(20.66 \%)$ & $2408(20.89 \%)$ & $3100(20.50 \%)$ & $2670(20.38 \%)$ & \\
\hline$\$ 50,000-\$ 74,999$ & $3809(21.13 \%)$ & $2515(21.82 \%)$ & $3447(22.79 \%)$ & $2962(22.61 \%)$ & \\
\hline$\geq \$ 75,000$ & $4062(22.53 \%)$ & $2674(23.20 \%)$ & $3651(24.14 \%)$ & $3304(25.23 \%)$ & \\
\hline \multicolumn{6}{|l|}{ Education } \\
\hline None-HS diploma & $4040(21.01 \%)$ & $2217(18.11 \%)$ & $2507(15.84 \%)$ & $2066(15.12 \%)$ & \multirow{3}{*}{$<.0001$} \\
\hline School after HS & $7050(36.67 \%)$ & $4335(35.41 \%)$ & $5557(35.10 \%)$ & $4685(34.28 \%)$ & \\
\hline College degree or higher & $8135(42.31 \%)$ & $5689(46.47 \%)$ & $7768(49.07 \%)$ & $6917(50.61 \%)$ & \\
\hline \multicolumn{6}{|l|}{ Physical activity } \\
\hline $\begin{array}{l}\text { Inactive (no reported } \\
\text { recreational activity) }\end{array}$ & $1971(10.26 \%)$ & $1250(10.24 \%)$ & $1815(11.46 \%)$ & $2243(16.42 \%)$ & \multirow[t]{4}{*}{$<.0001$} \\
\hline <5 MET-hrs/week & $3026(15.75 \%)$ & $2066(16.92 \%)$ & $2851(18.01 \%)$ & $2871(21.02 \%)$ & \\
\hline 5-<12 MET-hrs/week & $4251(22.12 \%)$ & $2830(23.18 \%)$ & $3942(24.90 \%)$ & $3382(24.76 \%)$ & \\
\hline$\geq 12$ MET-hrs/week & $9967(51.87 \%)$ & $6064(49.66 \%)$ & $7223(45.63 \%)$ & $5162(37.79 \%)$ & \\
\hline \multicolumn{6}{|l|}{ Self-rate health status } \\
\hline Excellent & $4366(22.62 \%)$ & $2545(20.72 \%)$ & $3312(20.79 \%)$ & $2587(18.83 \%)$ & \multirow{4}{*}{$<.0001$} \\
\hline Very good & $8399(43.51 \%)$ & $5552(45.21 \%)$ & $6984(43.84 \%)$ & $5764(41.96 \%)$ & \\
\hline Good & $5324(27.58 \%)$ & $3525(28.71 \%)$ & $4624(29.03 \%)$ & $4188(30.48 \%)$ & \\
\hline Fair/poor & $1214(6.29 \%)$ & $658(5.36 \%)$ & $1009(6.33 \%)$ & $1199(8.73 \%)$ & \\
\hline \multicolumn{6}{|l|}{ BMI } \\
\hline$<18.5$ & $260(1.36 \%)$ & $135(1.11 \%)$ & $157(0.99 \%)$ & $112(0.82 \%)$ & \multirow{4}{*}{$<.0001$} \\
\hline $18.5-24.9$ & $8655(45.20 \%)$ & $5389(44.27 \%)$ & $6450(40.87 \%)$ & $5007(36.83 \%)$ & \\
\hline $25-29.9$ & $6646(34.70 \%)$ & $4112(33.78 \%)$ & $5520(34.98 \%)$ & $4464(32.83 \%)$ & \\
\hline$>30.0$ & 3589 (18.74\%) & $2536(20.83 \%)$ & $3655(23.16 \%)$ & $4013(29.52 \%)$ & \\
\hline \multicolumn{6}{|l|}{ Smoking } \\
\hline Never & $10296(53.85 \%)$ & $6323(51.92 \%)$ & $7874(49.84 \%)$ & $6425(47.14 \%)$ & \multirow[t]{3}{*}{$<.0001$} \\
\hline Past & $7873(41.18 \%)$ & $5258(43.17 \%)$ & $7127(45.12 \%)$ & $6380(46.81 \%)$ & \\
\hline Current & $951(4.97 \%)$ & $598(4.91 \%)$ & $796(5.04 \%)$ & $825(6.05 \%)$ & \\
\hline
\end{tabular}


TABle 1: Continued.

\begin{tabular}{|c|c|c|c|c|c|}
\hline \multirow{3}{*}{ Characteristic } & \multicolumn{4}{|c|}{ Total sedentary time (hours per day) ${ }^{1}$} & \multirow{3}{*}{$P$ value $^{2}$} \\
\hline & Q1 ( $\leq 6 \mathrm{hrs} /$ day $)$ & Q2 (>6-8 hrs/day) & Q3 (>8-11 hrs/day) & Q4 (>11 hrs/day) & \\
\hline & $n(\%)$ & $n(\%)$ & $n(\%)$ & $n(\%)$ & \\
\hline \multicolumn{6}{|l|}{ Alcohol intake, per week } \\
\hline $0 /$ past drinker & $5456(28.34 \%)$ & $3078(25.08 \%)$ & $3793(23.86 \%)$ & $3381(24.66 \%)$ & \multirow{4}{*}{$<.0001$} \\
\hline$<1$ & $5966(30.99 \%)$ & $3851(31.38 \%)$ & $5180(32.59 \%)$ & $4732(34.51 \%)$ & \\
\hline $1-14$ & $6961(36.16 \%)$ & $4769(38.85 \%)$ & $6134(38.59 \%)$ & $4939(36.02 \%)$ & \\
\hline$\geq 14$ & $866(4.50 \%)$ & $576(4.69 \%)$ & $789(4.96 \%)$ & $661(4.82 \%)$ & \\
\hline \multicolumn{6}{|l|}{ Hormone use } \\
\hline Never used & $5485(28.83 \%)$ & $3452(28.55 \%)$ & $4304(27.46 \%)$ & $3526(26.10 \%)$ & \multirow{3}{*}{$<.0001$} \\
\hline Past user & $3811(20.03 \%)$ & $2382(19.70 \%)$ & $3127(19.95 \%)$ & $2656(19.66 \%)$ & \\
\hline Current user & $9729(51.14 \%)$ & $6257(51.75 \%)$ & $8245(52.60 \%)$ & $7326(54.23 \%)$ & \\
\hline \multicolumn{6}{|l|}{ Depressed mood } \\
\hline 0 & $5906(31.00 \%)$ & $3442(28.26 \%)$ & $4068(25.70 \%)$ & $3047(22.36 \%)$ & \multirow{4}{*}{$<.0001$} \\
\hline $1-2$ & $7137(37.46 \%)$ & $4840(39.74 \%)$ & $6183(39.06 \%)$ & $5191(38.09 \%)$ & \\
\hline $3-4$ & $3670(19.26 \%)$ & $2355(19.33 \%)$ & $3297(20.83 \%)$ & $2955(21.68 \%)$ & \\
\hline $5+$ & $2340(12.28 \%)$ & $1543(12.67 \%)$ & $2282(14.42 \%)$ & $2437(17.88 \%)$ & \\
\hline Living alone & $4025(20.90 \%)$ & $2815(22.96 \%)$ & $4028(25.34 \%)$ & $3963(28.92 \%)$ & $<.0001$ \\
\hline $\begin{array}{l}\text { Activity of daily living disability } \\
\text { ( } \geq 1 \text { disability) }\end{array}$ & $238(1.27 \%)$ & $139(1.16 \%)$ & $200(1.29 \%)$ & $281(2.10 \%)$ & $<.0001$ \\
\hline History of $\mathrm{CHD}^{3}$ & $1098(5.77 \%)$ & $759(6.24 \%)$ & $958(6.09 \%)$ & $789(5.82 \%)$ & 0.2638 \\
\hline History of CHF & $159(0.84 \%)$ & $99(0.82 \%)$ & $156(1.00 \%)$ & $126(0.93 \%)$ & 0.3204 \\
\hline History of stroke & $217(1.12 \%)$ & $126(1.02 \%)$ & $159(1.00 \%)$ & $144(1.05 \%)$ & 0.6934 \\
\hline $\begin{array}{l}\text { History of diabetes (use of pills } \\
\text { or shots) }\end{array}$ & $500(2.59 \%)$ & $295(2.40 \%)$ & $439(2.75 \%)$ & $442(3.21 \%)$ & 0.0003 \\
\hline $\begin{array}{l}\text { Hypertensive (on medications } \\
\text { for high blood pressure) }\end{array}$ & $6826(35.64 \%)$ & $4340(35.61 \%)$ & $5594(35.39 \%)$ & $4715(34.61 \%)$ & 0.2261 \\
\hline History of arthritis & $8672(45.11 \%)$ & $5852(47.78 \%)$ & $7590(47.85 \%)$ & $6577(47.99 \%)$ & $<.0001$ \\
\hline History of cancer & $2255(11.73 \%)$ & $1513(12.36 \%)$ & $1954(12.32 \%)$ & $1728(12.61 \%)$ & 0.0826 \\
\hline History of COPD ${ }^{3}$ & $533(2.81 \%)$ & $349(2.89 \%)$ & $507(3.23 \%)$ & $518(3.83 \%)$ & $<.0001$ \\
\hline \multicolumn{6}{|l|}{$\begin{array}{l}\text { Number of falls in the previous } \\
12 \text { months }\end{array}$} \\
\hline None & 13245 (69.14\%) & $8353(68.47 \%)$ & $10530(66.64 \%)$ & $8924(65.44 \%)$ & \multirow{4}{*}{$<.0001$} \\
\hline 1 time & $3741(19.53 \%)$ & $2436(19.97 \%)$ & $3254(20.59 \%)$ & $2867(21.03 \%)$ & \\
\hline 2 times & $1467(7.66 \%)$ & $928(7.61 \%)$ & $1372(8.68 \%)$ & $1212(8.89 \%)$ & \\
\hline 3 or more times & $703(3.67 \%)$ & $482(3.95 \%)$ & $646(4.09 \%)$ & $633(4.64 \%)$ & \\
\hline $\begin{array}{l}\text { History of hip fracture at age } \\
\geq 55 \text { years }\end{array}$ & $83(0.54 \%)$ & $70(0.72 \%)$ & $77(0.63 \%)$ & $67(0.68 \%)$ & 0.3034 \\
\hline \multicolumn{6}{|l|}{ Number of chronic diseases } \\
\hline 0 & $4515(23.32 \%)$ & $2699(21.91 \%)$ & $3406(21.34 \%)$ & $2934(21.31 \%)$ & \multirow{6}{*}{$<.0001$} \\
\hline 1 & $6441(33.26 \%)$ & $4041(32.81 \%)$ & $5181(32.47 \%)$ & $4357(31.64 \%)$ & \\
\hline 2 & $4661(24.07 \%)$ & $3065(24.89 \%)$ & $3955(24.78 \%)$ & $3391(24.63 \%)$ & \\
\hline 3 & $2336(12.06 \%)$ & $1532(12.44 \%)$ & $2074(13.00 \%)$ & $1819(13.21 \%)$ & \\
\hline 4 & $932(4.81 \%)$ & $656(5.33 \%)$ & $876(5.49 \%)$ & $850(6.17 \%)$ & \\
\hline $5+$ & $479(2.47 \%)$ & $323(2.62 \%)$ & $466(2.92 \%)$ & $418(3.04 \%)$ & \\
\hline
\end{tabular}

${ }^{1}$ All WHI-OS participants who reported on sitting time and sleeping time at baseline were enrolled in the extension study and had filled out at least one Form $151(N=61,609)$. All the percentage calculations were based on number of participants who reported on the individual variable.

${ }^{2} P$ value was based on chi-square test for the null hypothesis of no overall difference in the baseline variable among the 4 groups.

${ }^{3}$ Coronary heart disease (CHD) included MI, angina, CABG, and PTCA. 
TABLE 2: Change in daily sitting time and total sedentary time between baseline and Year 3 of the OS, stratified by age.

\begin{tabular}{lcccc}
\hline Characteristic & All $(N$, mean $(\mathrm{SD}))$ & $50-59(N$, mean $(\mathrm{SD}))$ & $\begin{array}{c}\text { Age Categories (years) } \\
60-69(N, \text { mean }(\mathrm{SD}))\end{array}$ & $70-79(N, \mathrm{mean}(\mathrm{SD}))$ \\
\hline Sitting time (hours/day) & & & & \\
$\quad$ Baseline & $61609,7.47(3.12)$ & $21363,8.07(3.35)$ & $27953,7.23(3.00)$ & $12293,6.97(2.79)$ \\
$\quad$ Year 3 & $58111,7.04(2.93)$ & $19888,7.57(3.20)$ & $26541,6.78(2.77)$ & $11682,6.71(2.67)$ \\
Change & $58111,-0.43(2.88)$ & $19888,-0.50(3.00)$ & $26541,-0.45(2.84)$ & $11682,-0.27(2.75)$ \\
$P$ value for change & $<.0001$ & $<.0001$ & $<.0001$ & $<.0001$ \\
\hline
\end{tabular}

Total sedentary time

(hours/day)

Baseline

$61407,8.63(3.82)$

$57565,8.09(3.44)$

Year 3

Change

$57390,-0.54$ (3.62)

$P$ value for change ${ }^{4}$
21303, 9.14 (3.94)

19697, 8.52 (3.62)

$19649,-0.61$ (3.63)
27860, 8.42 (3.74)

26308, 7.85 (3.33)

$26225,-0.58(3.60)$
12244, 8.21 (3.67)

11560, $7.88(3.30)$

$11516,-0.33$ (3.64)

${ }^{4} P$ value was based on a 1 -df $t$-test for the hypothesis of no significant change between baseline and year.

based on Chi-Square tests. Values for sitting time and total sedentary time at baseline and year 3 along with change between those time points, stratified by age, are examined to determine the amount of change in these behaviors.

Linear mixed model analysis of repeated measures was used to estimate mean PF scores across follow-up time in relation to sedentary time exposures, adjusted for the baseline covariates mentioned above. This method first attempted to estimate the covariance structure of PF scores across various follow up times by using both graphical tools and the information criteria statistics. The first-order autoregressive [AR (1)] covariance structure was chosen to best represent the correlation structure. Then, the mixed model assessed the relationship between sedentary time exposures and change in PF scores using generalized least squares with the estimated covariance. To fully utilize the data source, the model updated the sedentary time exposures at Year 3 when available to incorporate the change in exposure variables.

To assess to what degree the association between sedentary behavior and decline in mean PF scores was affected by physical activity level, the interaction between baseline sedentary time and baseline physical activity as well as the interaction between year 3 sedentary time and year 3 physical activity on PF scores were evaluated in separate models, adjusting for the aforementioned set of covariates. In addition, to account for new onset of comorbidity (CHD, CHF, cancer, stroke, diabetes, hypertensive, and hip fracture over age 55) that occurred after year 3, secondary analyses repeated the primary analyses with adjustment for new onset of comorbidity. All statistical analyses were performed using SAS statistical software (version 9.2; SAS Institute Inc, Cary, NC, USA).

\section{Results}

Participant characteristics by quartile of total sedentary time $(\leq 6,6-8,8-11,>11$ hours/day) at baseline are shown in Table 1 . Women within the first three quartiles were similarly distributed across age groups, with a modestly smaller percentage of 70-79-year olds in the fourth quartile. Women from minority race/ethnic groups were more likely to report the least sedentary time. Trends in education and income also showed higher percentages of the most highly educated and those with more income having greater amounts of the sedentary time. Women reporting less sedentary time reported higher physical activity levels and higher selfreported health status. They also had lower prevalence of obesity (BMI $\geq 30)$ and reported less-frequent depressed mood. Women with higher sedentary time reported a greater number of chronic diseases overall, although there were no differences in the prevalence of many specific diseases examined across quartiles of sedentary time. Women with greater sedentary time also reported a higher frequency of falls over the past 12 months, activity of daily living (ADL) disability, and were more likely to live alone.

Reported sedentary time at baseline and at year 3 were moderately correlated (Pearson $r=0.55$ ). On average, women reported about a half-hour less of sitting time and total sedentary time at year 3 compared to baseline (Table 2). The greatest reductions occurred among the 50-59-year olds $(-0.60$ hours $)$ and $60-69$-year olds ( -0.57 hours). This equates, approximately, to a reduction of 35 minutes of sedentary time per day among the 50-59- and 60-69-year olds and to a reduction of 20 minutes per day among the 70-79-year olds.

After adjustment for covariates, greater amounts of sitting time and total sedentary time were significantly associated with lower mean PF scores (Table 3). Compared to those who reported sedentary time of $\leq 6$ hours/day, those with greater amounts of sedentary time ( $>6-8$ hours/day, $>8-11$ hours/day, and $>11$ hours/day) had lower PF scores between baseline and approximately 12 years of follow up [coefficient $=-0.78,95 \%$ confidence interval $(\mathrm{CI})=-0.98$, $-0.57,-1.48, \mathrm{CI}=-1.71,-1.25,-3.13, \mathrm{CI}=-3.36,-2.89$, resp.; $P<0.001]$. Similar associations were seen across quartiles of sitting time and PF scores at follow up (coefficient $=$ $-0.96, \mathrm{CI}=-1.16,-0.76 ;-1.45, \mathrm{CI}=-1.68,-1.22,-2.45$, $\mathrm{CI}=-2.69,-2.22$, resp.; $P<0.001)$. Thus, in examining 
TABLE 3: Multivariate analysis of change in physical function score over time between WHI-OS baseline and WHI-OSES follow up and daily sitting time and total sedentary time ${ }^{5}$.

\begin{tabular}{lcc}
\hline Key variable & & Change in mean physical function score \\
& Parameter estimate & $95 \%$ CI \\
\hline Sitting time at baseline & & \\
Q1 ( $\leq 4.5$ hours/day) & 1.00 & $($ referent $)$ \\
Q2 (>4.5-6.5 hours/day) & -0.96 & $(-1.16,-0.76)$ \\
Q3 (>6.5-8.5 hours/day) & -1.45 & $(-1.68,-1.22)$ \\
Q4 (>8.5 hours/day) & -2.45 & $(-2.69,-2.22)$ \\
\hline Total sedentary time at baseline & & $($ referent $)$ \\
Q1 ( $\leq 6$ hours/day) & 1.00 & $(-0.98,-0.57)$ \\
Q2 (>6-8 hours/day) & -0.78 & $(-1.71,-1.25)$ \\
Q3 (>8-11 hours/day) & -1.48 & $(-3.36,-2.89)$ \\
Q4 (>11 hours/day) & -3.13 & $<.0001$ \\
\hline
\end{tabular}

${ }^{5}$ Results from linear mixed model of repeated measures assuming AR (1) covariance structure, adjusted for age race/ethnicity, education, physical activities, self-reported general health, BMI, smoking status, alcohol use, hormone use, depressed mood, living alone, activities of daily living, history of CHD, history of CHF, stroke, treated diabetes, arthritis, and history of COPD at baseline. Key variables were estimated in separated models and updated in the model with Year 3 data when available.

the relationships for total sedentary time, women reporting $>11$ hours per day had more than three times lower mean PF scores compared to women in the lowest category ( $\leq 6$ hours per day), with comparable findings for sitting time, as shown in Table 3. The secondary analyses that accounted for new onset of comorbidity after year 3 revealed similar relationships (data not shown).

The interaction between baseline sedentary time and baseline physical activities on PF scores was borderline significant (interaction $P=0.0575$ ). However, the association of year 3 sedentary time with change in mean PF scores differed by level of physical activity at year 3 (interaction $P<0.0001)$. Compared to women who were inactive and reported sedentary time of $\leq 5$ hours/day at year 3 , women who reported higher levels of physical activity and the same amount of sedentary time ( $\leq 5$ hours/day) experienced less decline in PF. Figures 1(a) and 1(b) display physical function score over time by baseline sitting time and baseline total sedentary time, respectively. Figures $2(a)-2(d)$ show sedentary time quartiles across four levels of physical activity.

\section{Discussion}

This paper contributes to a growing body of literature that supports the notion that sedentary time behavior may well be a distinct component of physical inactivity not merely reflecting an adverse exposure at the extreme low end of the physical activity continuum. We found that women reporting the largest amounts of sedentary time had higher PF scores compared to those reporting less sedentary time. This association was dose-responsive and independent of numerous potential confounding variables including self-reported physical activity from vigorous, moderate, and mild activities as well as walking.

In addition, these findings not only demonstrate independent associations between total sedentary time and physical activity, but also show an interaction between the two activity variables. Greater amounts of physical activity were clearly related to higher PF scores. Nonetheless, in every category of physical activity, declines in PF were greatest in the women reporting the most sedentary time. Women with the least physical activity and the most sedentary time had the most precipitous drops in PF. Thus, even in the context of varying levels of physical activity, these data support the deleterious effects of time spent in sedentary activities. It would be beneficial for future research to attempt to disaggregate the separate contributions of physical activity and sedentary time on health outcomes related to maintaining independence in later life.

In a recent study, light-intensity physical activity, measured objectively with an accelerometer, was significantly associated with better physical health, including lower extremity function, as well as other general health benefits among adults older than 65 years [20]. Although there are still considerations related to defining physical activity intensity using accelerometer cut-points in older adults, these data show health benefits, including higher PF associated with light activity. Avoiding sedentary activities may therefore confer important health benefits for some individuals even at activity levels below recommendations and supports the contention that some activity is better than none [6].

The present study also showed interesting trends related to sedentary time that may be partly explained by occupation and other socioeconomic variables. Contrary to other research that shows associations between obesity (and obesity-related chronic diseases) and low-income, less educated populations [28], these data tell a different story. Trends in education and income show higher percentages of the most highly educated and those with more income having greater amounts of sedentary time. This may be explained by occupation, number of work hours per day, and/or the ability to afford assistance with household chores, such as cleaning or yard work. 


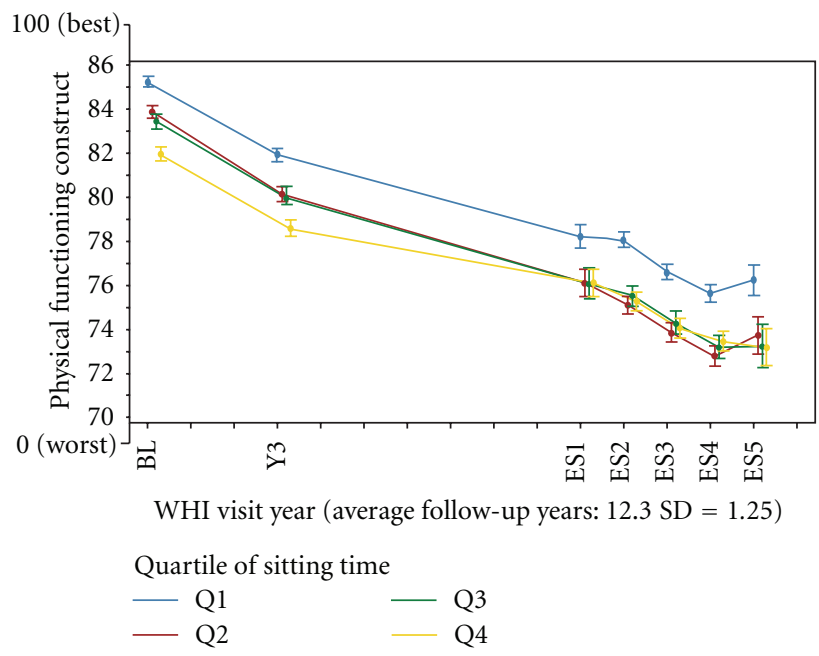

(a)

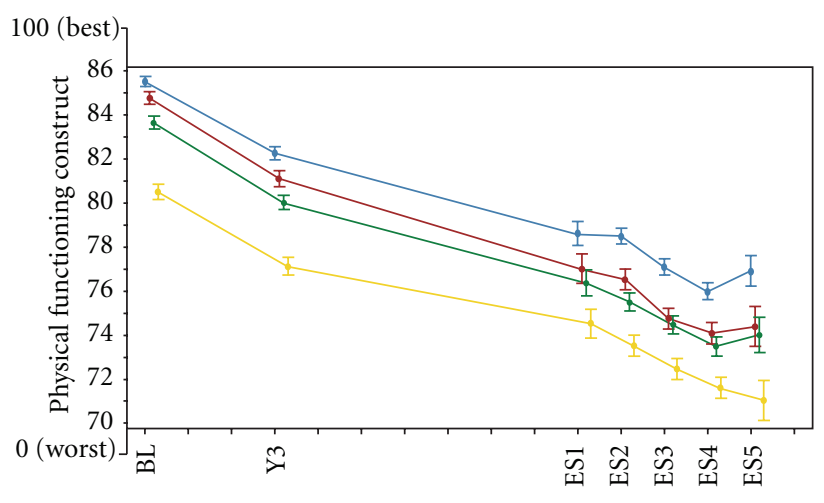

WHI visit year (average follow-up years: $12.3 \mathrm{SD}=1.25$ )

Quartile of total sedentary time

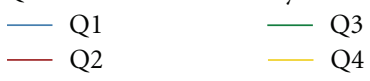

(b)

Figure 1: (a) Physical function score over time by baseline sitting time, $N=61,609$. (b) Physical function score over time by baseline total sedentary time, $N=61,609$.

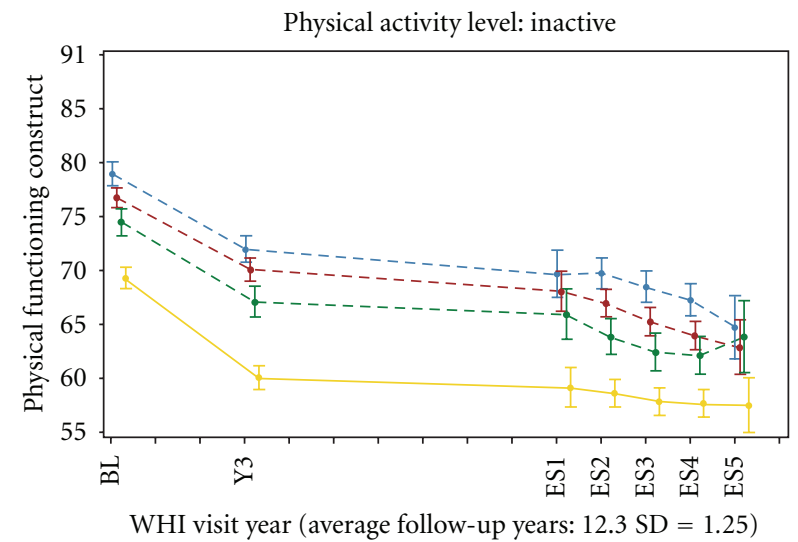

(a)

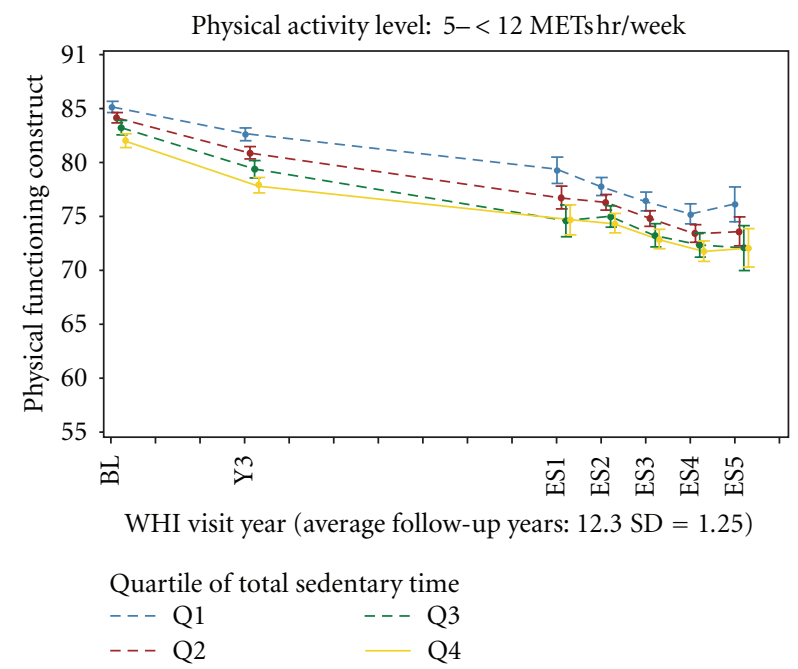

(c)

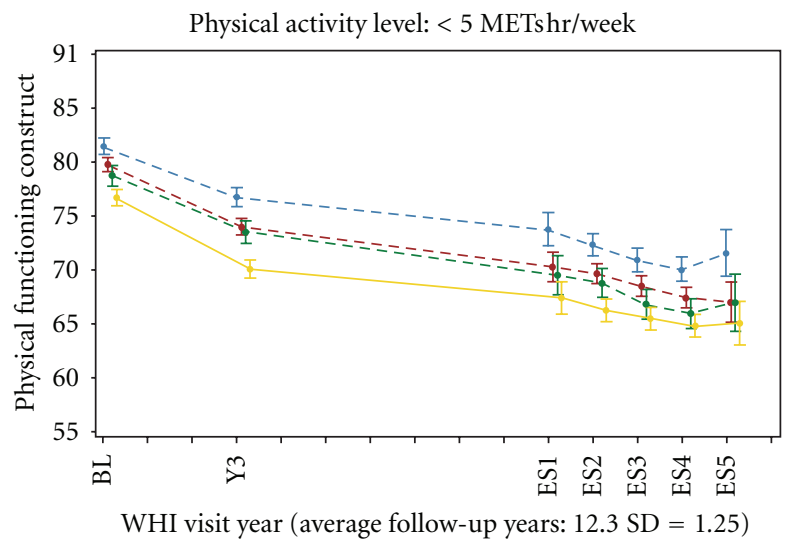

(b)

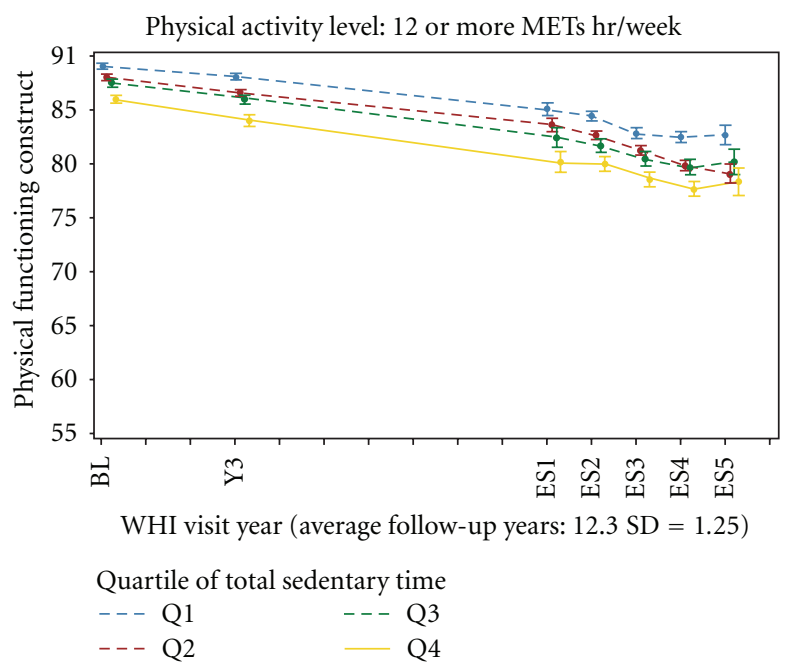

(d)

Figure 2: Physical function score over time by total sedentary time (year 3) and physical activity level, $N=61,609$. 
There may also be occupational factors differentiating between sedentary hours and age. Between baseline and year 3 , the greatest reductions in sedentary time occurred among the 50-69 year olds, which was approximately thirtyfive minutes per day. Comparatively, women in the eldest age group reduced sedentary time by approximately twenty minutes per day. This trend among younger women may be related to entry into retirement, leading to less time spent doing seated tasks, such as computer work, and more time engaged in light, household, or leisure activities that may not always be captured as "physical activity," especially when assessed by questionnaire. More active women also tended to not live alone, indicating that cohabitation is associated with less daily sedentary time and pointing to the potentially positive influence of social relationships in facilitating better health behaviors.

These data are consistent with the hypothesis that participating in basic activities on a regular basis (e.g., simple household chores) could be a metabolic stimulus that helps to preserve ADLs and independence with aging. This may be especially true in the older subgroup of the elderly population, through preserving PF including skeletal muscle regulatory function. It is also important to note, however, that many self-reported physical activity questionnaires do not specifically ask about "light" activity, which may be quantified by the difference between moderate or vigorous activity and sedentary time. This research has important public health implications, given that declines in physical performance (strength, balance, and mobility) are associated with disability, morbidity, nursing home admissions, and other adverse consequences [29] —all of which confer substantial medical costs.

Strengths of this study include the large sample size, inclusion of women diverse in race/ethnicity and socioeconomic status, more than ten years of prospective follow up on $\mathrm{PF}$, and volume of data on potential confounders including the occurrence of incident disease. The findings are limited by not having objective measurements of sedentary time and not having a physical activity questionnaire that fully captured light intensity activity. This study was observational and does not establish a causal relationship between sedentary time and loss of physical function. Experimental studies are needed to test interventions that reduce sedentary time, and that explicate the role of relatively light intensity at lower than recommended volumes in preserving PF.

The contribution of these data relates to both policy and clinical practice. First, expanding public health messages to reduce sedentary time and increase activity levels are likely to impart the broadest health benefits. Targeted messages to reduce time spent engaged in sedentary activities may have potential for impact when paired with current physical activity recommendations for older adults. Further, in conjunction with other findings $[6,30]$, these data provide a platform for clinicians to communicate with older patients about the benefits of general household and daily activities, irrespective of participation in a regular physical activity program. Although formal exercise participation is optimal, there appears to be a clear benefit of reducing sedentary activities among aging women that may confer extended duration of independent living and improved quality of life.

\section{Authors' Contribution}

All authors contributed significantly to preparation of the paper.

\section{Conflict of Interests}

The authors have no conflict of interests to declare.

\section{Acknowledgments}

The authors acknowledge Program Office: National Heart, Lung, and Blood Institute, Bethesda, MD (Elizabeth Nabel, Jacques Rossouw, Shari Ludlam, Joan McGowan, Leslie Ford, and Nancy Geller); Clinical Coordinating Center: Fred Hutchinson Cancer Research Center, Seattle, WA (Ross Prentice, Garnet Anderson, Andrea LaCroix, Charles L. Kooperberg, Ruth E. Patterson, and Anne McTiernan); Medical Research Labs, Highland Heights, Kentucky: Evan Stein. University of California at San Francisco, San Francisco, CA (Steven Cummings); Clinical Centers: Albert Einstein College of Medicine, Bronx, NY (Sylvia WassertheilSmoller); Baylor College of Medicine, Houston, TX (Aleksandar Rajkovic); Brigham and Women's Hospital, Harvard Medical School, Boston, MA (JoAnn E. Manson); Brown University, Providence, Rhode Island: Charles B. Eaton, Emory University, Atlanta, GA (Lawrence Phillips); Fred Hutchinson Cancer Research Center, Seattle, WA (Shirley Beresford); George Washington University Medical Center, WA, DC (Lisa Martin); Los Angeles Biomedical Research Institute at Harbor-UCLA Medical Center, Torrance, CA (Rowan Chlebowski); Kaiser Permanente Center for Health Research, Portland, OR (Yvonne Michael); Kaiser Permanente Division of Research, Oakland, CA (Bette Caan); Medical College of Wisconsin, Milwaukee, WI (Jane Morley Kotchen); MedStar Research Institute, Howard University, WA, DC (Barbara V. Howard); Northwestern University, Chicago/Evanston, IL (Linda Van Horn); Rush Medical Center, Chicago, IL (Henry Black); Stanford Prevention Research Center, Stanford, CA (Marcia L. Stefanick); State University of New York at Stony Brook, Stony Brook, NY (Dorothy Lane); The Ohio State University, Columbus, OH (Rebecca Jackson); University of Alabama at Birmingham, Birmingham, AL (Cora E. Lewis); University of Arizona, Tucson, Phoenix, AZ (Cynthia A. Thomson); University at Buffalo, Buffalo, NY (Jean Wactawski- Wende); University of California at Davis, Sacramento, CA (John Robbins); University of California at Irvine, CA (F. Allan Hubbell); University of California at Los Angeles, Los Angeles, CA (Lauren Nathan); University of California at San Diego, La Jolla/Chula Vista, CA (Robert D. Langer); University of Cincinnati, Cincinnati, OH (Margery Gass); University of Florida, Gainesville/Jacksonville, FL (Marian Limacher); University of Hawaii, Honolulu, HI (J. David Curb); University of Iowa, Iowa City/Davenport, IA (Robert Wallace); University of Massachusetts, Fallon Clinic, Worcester, MA 
(Judith Ockene); University of Medicine and Dentistry of New Jersey, Newark, NJ (Norman Lasser); University of Miami, Miami, FL (Mary Jo O'Sullivan); University of Minnesota, Minneapolis, MN (Karen Margolis); University of Nevada, Reno, NV (Robert Brunner); University of North Carolina, Chapel Hill, NC (Gerardo Heiss); University of Pittsburgh, Pittsburgh, PA (Lewis Kuller); University of Tennessee Health Science Center, Memphis, TN (Karen C. Johnson); University of Texas Health Science Center, San Antonio, TX (Robert Brzyski); University of Wisconsin, Madison, WI, (Gloria E. Sarto); Wake Forest University School of Medicine, Winston-Salem, NC (Mara Vitolins); Wayne State University School of Medicine, Hutzel Hospital, Detroit, MI (Michael Simon). The WHI Program is funded by the National Heart, Lung, and Blood Institute (NHLBI), National Institutes of Health, US Department of Health and Human Services through Contracts N01WH22110, 24152, 32100-2, 32105-6, 32108-9, 32111-13, 32115, 32118-32119, $32122,42107-26,42129-32$, and 44221. This work was also supported by PO1 CA53996, R01AG025441-03, and T32 AG027677. R. Seguin is supported by the T32 AG027677 fellowship sponsored by the National Institute of Aging. A. LaCroix was supported by 5R01AG025441-03 sponsored by the National Institute of Aging. M. LaMonte was supported by HHSN26820110001C sponsored by the National Heart Lung and Blood Institute. L. Tinker was supported by N01WH-2-2110 sponsored by the National Heart Lung and Blood Institute. J. Liu was supported by N01-WH-2-2110 sponsored by the National Heart Lung and Blood Institute. J. Liu had full access to all of the data in the study and takes responsibility for the integrity of the data and the accuracy of the data analysis. Y. Michael was supported by R03AG031973 sponsored by the National Institute of Aging. C. Bushnell was supported by NIH K02 NS058760 sponsored by the National Institute for Neurological Disorders and Stroke.

\section{References}

[1] J. E. Manson, P. Greenland, A. Z. LaCroix et al., "Walking compared with vigorous exercise for the prevention of cardiovascular events in women," The New England Journal of Medicine, vol. 347, no. 10, pp. 716-725, 2002.

[2] P. D. Thompson, D. Buchner, I. L. Pina et al., "Exercise and physical activity in the prevention and treatment of atherosclerotic cardiovascular disease: a statement from the Council on Clinical Cardiology (Subcommittee on Exercise, Rehabilitation, and Prevention) and the Council on Nutrition, Physical Activity, and Metabolism (Subcommittee on Physical Activity)," Circulation, vol. 107, no. 24, pp. 3109-3116, 2003.

[3] M. E. Nelson, J. E. Layne, M. J. Bernstein et al., "The Effects of multidimensional home-based exercise on functional performance in elderly people," Journals of Gerontology A, vol. 59, no. 2, pp. 154-160, 2004.

[4] C. Castaneda, J. E. Layne, L. Munoz-Orians et al., "A randomized controlled trial of resistance exercise training to improve glycemic control in older adults with type 2 diabetes," Diabetes care, vol. 25, no. 12, pp. 2335-2341, 2002.

[5] M. E. Nelson, M. A. Fiatarone, C. M. Morganti, I. Trice, R. A. Greenberg, and W. J. Evans, "Effects of high-intensity strength training on multiple risk factors for osteoporotic fractures,"
Journal of the American Medical Association, vol. 272, no. 24, pp. 1909-1914, 1994.

[6] DHHS, Physical Activity Guidelines Advisory Committee Report, Department of Health and Human Services, Washington, DC, USA, 2008.

[7] S. A. Carlson, J. E. Fulton, D. A. Galuska, J. Kruger, F. Lobelo, and F. V. Loustalot, "Prevalence of self-reported physically active adults-United States, 2007," Morbidity and Mortality Weekly Report, vol. 57, no. 48, pp. 1297-1300, 2008.

[8] J. S. Brach, E. M. Simonsick, S. Kritchevsky, K. Yaffe, and A. B. Newman, "The Association between physical function and lifestyle activity and exercise in the health, aging and body composition study," Journal of the American Geriatrics Society, vol. 52, no. 4, pp. 502-509, 2004.

[9] K. R. Baker, M. E. Nelson, D. T. Felson, J. E. Layne, R. Sarno, and R. Roubenoff, "The efficacy of home based progressive strength training in older adults with knee osteoarthritis: a randomized controlled trial," Journal of Rheumatology, vol. 28, no. 7, pp. 1655-1665, 2001.

[10] N. A. Singh, K. M. Clements, and M. A. Fiatarone, "A randomized controlled trial of progressive resistance training in depressed elders," Journals of Gerontology A, vol. 52, no. 1, pp. M27-M35, 1997.

[11] N. Singh, K. Clements, and M. Fiatarone, "Sleep, sleep deprivation, and daytime activities: a randomized controlled trial of the effect of exercise on sleep," Sleep, vol. 20, pp. 95$101,1997$.

[12] M. V. Chakravarthy, M. J. Joyner, and F. W. Booth, "An obligation for primary care physicians to prescribe physical activity to sedentary patients to reduce the risk of chronic health conditions," Mayo Clinic Proceedings, vol. 77, no. 2, pp. 165$173,2002$.

[13] W. H. Dietz, L. G. Bandini, J. Morelli, K. F. Peers, and P. L. Y. H. Ching, "Effect of sedentary activities on resting metabolic rate," American Journal of Clinical Nutrition, vol. 59, no. 3, pp. 556-559, 1994.

[14] G. A. Colditz, "Economic costs of obesity and inactivity," Medicine and Science in Sports and Exercise, vol. 31, supplement 11, pp. S663-S667, 1999.

[15] L. Bey and M. T. Hamilton, "Suppression of skeletal muscle lipoprotein lipase activity during physical inactivity: a molecular reason to maintain daily low-intensity activity," Journal of Physiology, vol. 551, no. 2, pp. 673-682, 2003.

[16] N. Owen, G. N. Healy, C. E. Matthews, and D. W. Dunstan, "Too much sitting: the population health science of sedentary behavior," Exercise and Sport Sciences Reviews, vol. 38, no. 3, pp. 105-113, 2010.

[17] G. N. Healy, D. W. Dunstan, J. E. Shaw, P. Z. Zimmet, and N. Owen, "Beneficial associations of physical activity with 2-h but not fasting blood glucose in Australian adults: the AusDiab study," Diabetes Care, vol. 29, no. 12, pp. 2598-2604, 2006.

[18] G. N. Healy, D. W. Dunstan, J. Salmon et al., "Breaks in sedentary time: beneficial associations with metabolic risk," Diabetes Care, vol. 31, pp. 661-666, 2008.

[19] P. T. Katzmarzyk, T. S. Church, C. L. Craig, and C. Bouchard, "Sitting time and mortality from all causes, cardiovascular disease, and cancer," Medicine and Science in Sports and Exercise, vol. 41, no. 5, pp. 998-1005, 2009.

[20] M. P. Buman, E. B. Hekler, W. L. Haskell et al., "Objective light-intensity physical activity associations with rated health in older adults," American Journal of Epidemiology, vol. 172, no. 10, pp. 1155-1165, 2010.

[21] J. S. Brach, S. FitzGerald, A. B. Newman et al., "Physical activity and functional status in community-dwelling older 
women: a 14-year prospective study," Archives of Internal Medicine, vol. 163, no. 21, pp. 2565-2571, 2003.

[22] G. L. Anderson, J. Manson, R. Wallace et al., "Implementation of the Women's Health Initiative study design," Annals of Epidemiology, vol. 13, no. 9, supplement, pp. S5-S17, 2003.

[23] J. Hays, J. R. Hunt, F. A. Hubbell et al., "The Women's Health Initiative recruitment methods and results," Annals of Epidemiology, vol. 13, no. 9, supplement, pp. S18-S77, 2003.

[24] G. Anderson, S. Cummings, L. S. Freedman et al., "Design of the Women's Health Initiative clinical trial and observational study," Controlled Clinical Trials, vol. 19, no. 1, pp. 61-109, 1998.

[25] J. E. Ware Jr., "SF-36 health survey update," Spine, vol. 25, no. 24, pp. 3130-3139, 2000.

[26] J. E. Ware Jr. and C. D. Sherbourne, “The MOS 36-item shortform health survey (SF-36). I. conceptual framework and item selection," Medical Care, vol. 30, no. 6, pp. 473-483, 1992.

[27] B. E. Ainsworth, W. L. Haskell, M. C. Whitt et al., "Compendium of physical activities: an update of activity codes and MET intensities," Medicine and Science in Sports and Exercise, vol. 32, no. 9, supplement, pp. 498S-504S, 2000.

[28] DHHS, Preventing Obesity and Chronic Diseases Through Good Nutrition and Physical Activity, Department of Health and Human Services, Centers for Disease Control and Prevention (CDC), 2005.

[29] W. J. Rejeski, P. H. Brubaker, D. C. Goff Jr. et al., "Translating weight loss and physical activity programs into the community to preserve mobility in older, obese adults in poor cardiovascular health," Archives of Internal Medicine, vol. 171, no. 10, pp. 880-886, 2011.

[30] M. E. Nelson, W. J. Rejeski, S. N. Blair et al., "Physical activity and public health in older adults: recommendation from the American College of Sports Medicine and the American Heart Association," Medicine and Science in Sports and Exercise, vol. 39, no. 8, pp. 1435-1445, 2007. 


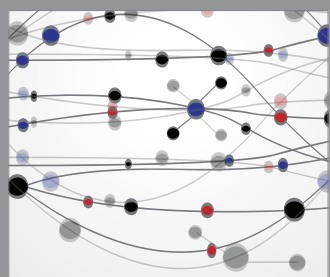

The Scientific World Journal
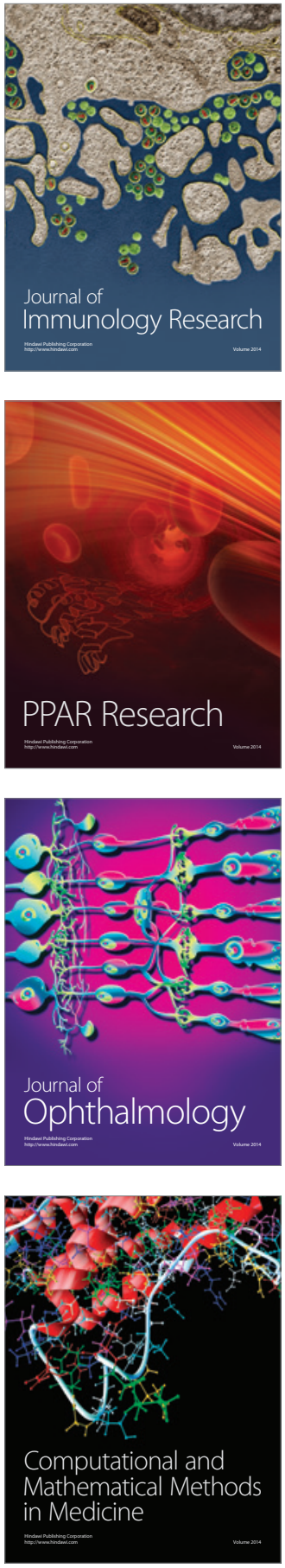

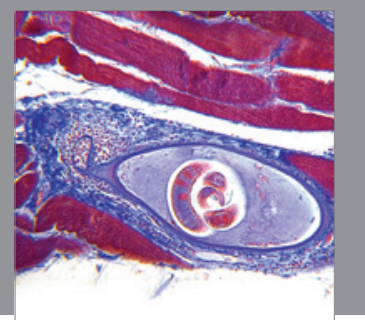

Gastroenterology

Research and Practice
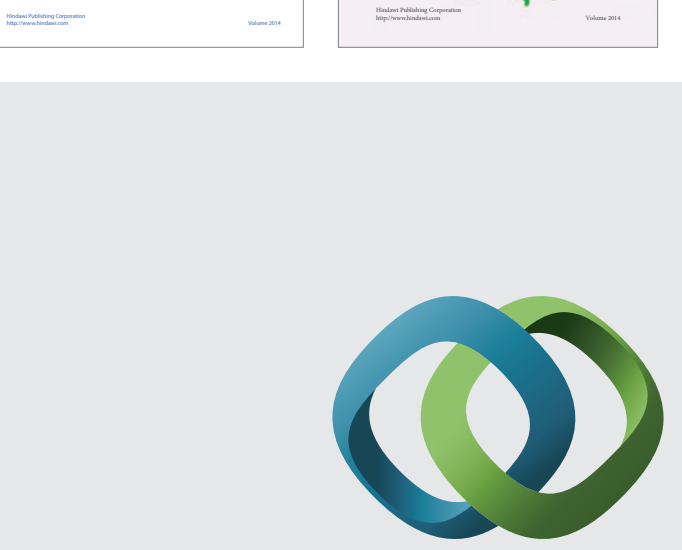

\section{Hindawi}

Submit your manuscripts at

http://www.hindawi.com
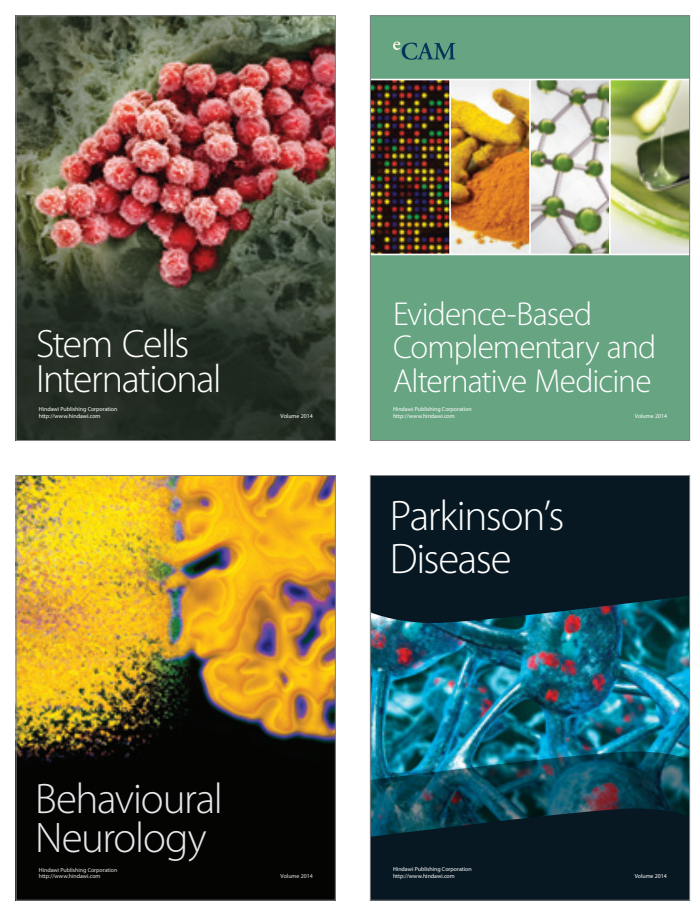

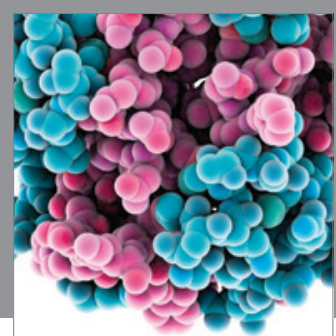

Journal of
Diabetes Research

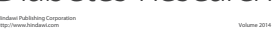

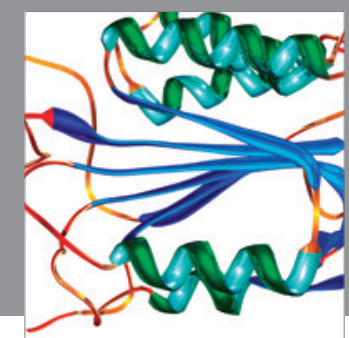

Disease Markers
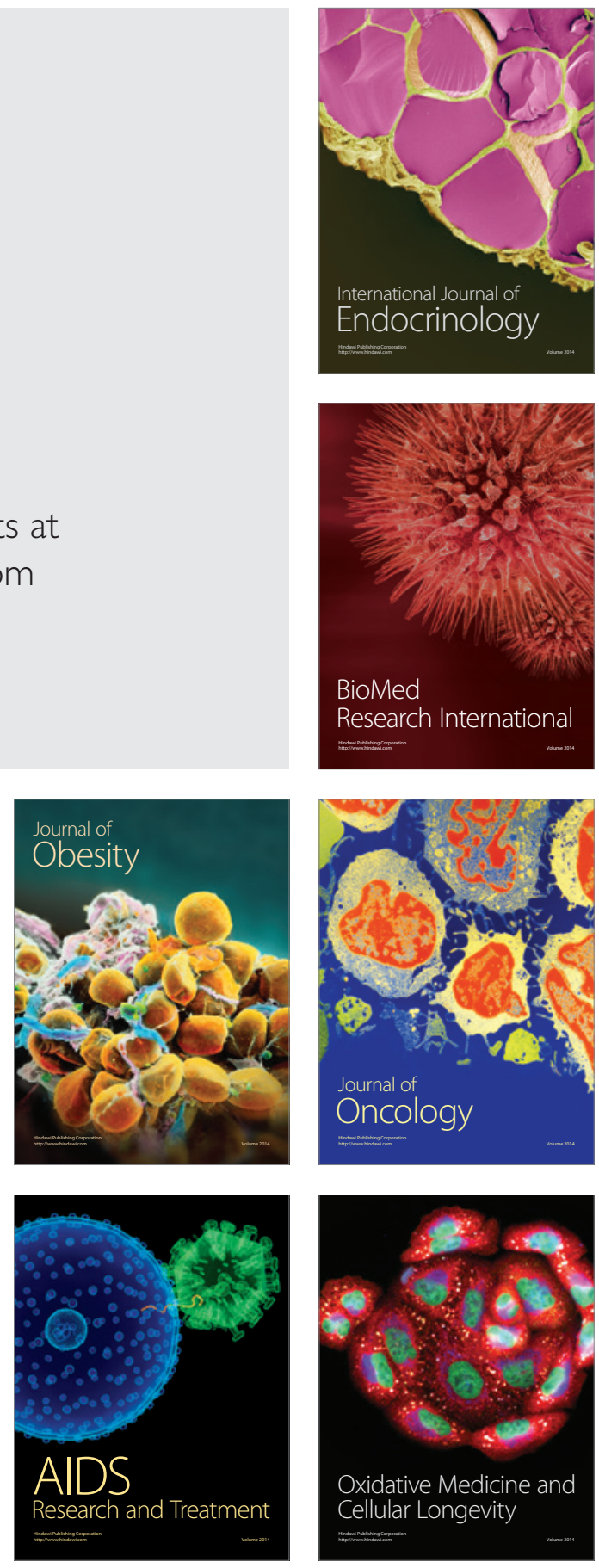